\title{
Explicit Authentication Response Considered Harmful ${ }^{*}$
}

\author{
Lianying Zhao and Mohammad Mannan \\ Concordia Institute for Information Systems Engineering \\ Concordia University, Montreal, Canada \\ \{z_lianyi,mmannan\}@encs.concordia.ca
}

\begin{abstract}
Automated online password guessing attacks are facilitated by the fact that most user authentication techniques provide a yes/no answer as the result of an authentication attempt. These attacks are somewhat restricted by Automated Turing Tests (ATTs, e.g., captcha challenges) that attempt to mandate human assistance. ATTs are not very difficult for legitimate users, but always pose an inconvenience. Several current ATT implementations are also found to be vulnerable to improved image processing algorithms. ATTs can be made more complex for automated software, but that is limited by the trade-off between user-friendliness and effectiveness of ATTs. As attackers gain control of large-scale botnets, relay the challenge to legitimate users at compromised websites, or even have ready access to cheap, sweat-shop human solvers for defeating ATTs, online guessing attacks are becoming a greater security risk. Using deception techniques (as in honeypots), we propose the user-verifiable authentication scheme (Uvauth) that tolerates, instead of detecting or counteracting, guessing attacks. Uvauth provides access to all authentication attempts; the correct password enables access to a legitimate session with valid user data, and all incorrect passwords lead to fake sessions. Legitimate users are expected to learn the authentication outcome implicitly from the presented user data, and are relieved from answering ATTs; the authentication result never leaves the server and thus remains (directly) inaccessible to attackers. In addition, we suggest using adapted distorted images and pre-registered images/text as a complement to convey an authentication response, especially for accounts that do not host much personal data.
\end{abstract}

\section{Categories and Subject Descriptors}

K.6.5 [Management of Computing and Information Systems]: Security and Protection-Authentication, Unauthorized access (e.g., hacking, phreaking)

\footnotetext{
*Pre-proceedings version: August 10, 2013
}

Permission to make digital or hard copies of all or part of this work for personal or classroom use is granted without fee provided that copies are not made or distributed for profit or commercial advantage and that copies bear this notice and the full citation on the first page. To copy otherwise, to republish, to post on servers or to redistribute to lists, requires prior specific permission and/or a fee.

NSPW'13, September 9-11, 2013, The Banff Centre, Canada

Copyright 2013 ACM xxx-X-xxx-Xxxx-x/xx/xx ...\$15.00.

\section{General Terms}

Security, Human Factors

\section{Keywords}

Authentication, Online password guessing, Deception

\section{INTRODUCTION}

Automated online pas: problem for password-bas problem is possibly getti following. (a) The growtl credentials; i.e., attacker tangible profits; see e.g., user accounts increases o book profiles, Gmail acc counts. In many cases, $\begin{array}{ll}\text { repla } & t \text { if the } \\ \text { old } c & \text { are not }\end{array}$ getti xity. New services requiring pass $\quad$ password fatigue or sharing acros number of online participant $c$ ommon passwords more lg more organized than jools and crackers; for e sbust botnets, and can 1 -forcing, e.g., opti-

Comn ber of the use

Pinkas tication phone, tackers extent. ing tech botnet (e.g., million-node) to test the most common passwords and possibly compr attacker is successful if her (e.g., to use as intermediat stead of compromising a t mostly detested by human creasingly difficult to deciph login times also increase as to try more than one cap eral real-world captcha sch proved image recognition -limiting the numn period of time; d attacks, see e.g., two-step authen-registered mobile tions. In most cases, atires, at least to a limited ree-strike account lock-

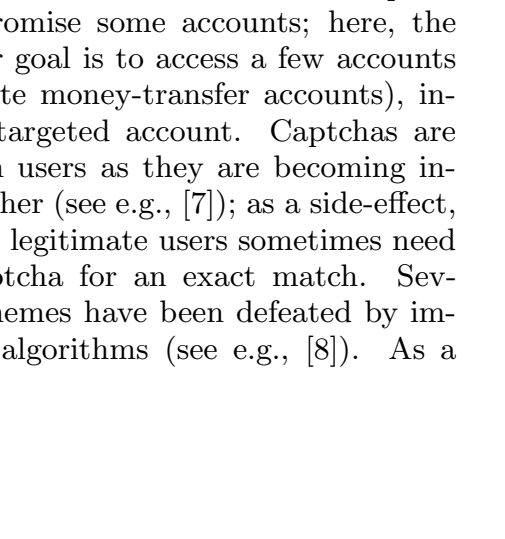


result, service providers often leave wit deploy more complex captchas. These lir and several proposals in the past attem security-usability trade

The fundamental pr tacker can learn the o tainty, using fully auto ial human help. Hum are available, for chea verifiers in all known a cess or failure message explicit messages aid o sages may include retu protocol data from the tinuation/discontinuation of the attempted session.

We introduce here Uvauth (user-verifiable authentication) to reduce the attacker's confidence on the outcome of her guessed password by granting her access for any password she enters. For a given userid, the correct password will lead to the real user account, and all other passwords will provide fake sessions (i.e., with fake user data). To avoid detection by re-logging into the same account, same useridpassword pair will always result in the same session. Likewise, different userid-password pairs should also lead to different sessions in order that the legitimate session cannot be distinguished from fake ones. The underlying assumption is that real users will implicitly understand the outcome of their authentication attempt by the presented data; i.e., an unfamiliar account will indicate that the entered password is incorrect, and they need to try again. On the other hand, a random attacker may have little or no idea what to expect as user data after being logged in, even if she launches a humanassisted attack. Attackers can perform different operations to discover a fake session, and our goal is to raise the bar for such attacks to succeed-e.g., by requiring non-trivial efforts from the attacker beyond simply solving a captcha.

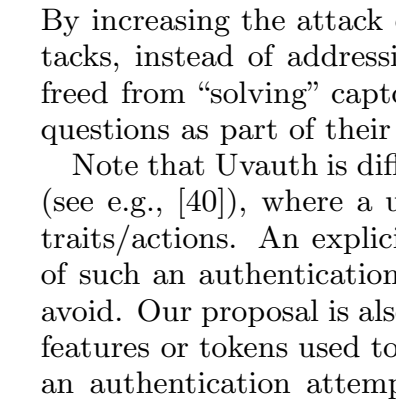
cost, we choose to tolerate the ating them head-on. Users are also chas, or answering personal secret authentication.

ferent than implicit authentication iser is authenticated by her usual it outcome is provided at the end attempt, which we would like to o independent of whatever secrets, verify a user; it i pt that we would where online guessing is a concern.

ns can be seen as a fo for centuries in trad warfare is based on ive defensive technolc any researchers for ye t computer security emonstrated in hone uence the behavior of use, e.g., to understal as and network resourc

ion is not to gain mo ors, but simply to rai tacks against weak au analogy may further
Consider a virtual building with several locked rooms. eypots protect access to a room by generating a fake on-the-fly or claiming that the room is unavailable. ntrast, we create a fake room to protect the lock of a , assuming the lock is weak - i.e., given enough time, k-picker can easily open it. Our use of fake sessions also be viewed as the no-information leakage property perfect one-time pad (OTP) encryption: attackers have vay of verifying a guessed key for an OTP scheme, as a valid key exists for every candidate plaintext, i.e., attackers do not know when they succeed.

Several challenges must be addressed for Uvauth. Generating fake sessions would require additional resources from the verifier, and non-trivial efforts to mimic a legitimate session. Protected accounts should have enough personal content so that legitimate users will easily learn whether they have logged in with the correct password. To address less/non-personal accounts, we propose the use of distorted images / modified captchas as a communication channel from the verifier to a client. The crucial difference with existing captcha here is that: we do not require users to solve captchas verbatim (i.e., character-by-character) and type the result. Instead, users are expected to use the captcha messages as a second channel to verify their login (i.e., in addition to the content they can see). More challenging captcha schemes can be used in our setting, as users are not required to decipher each character in the exact form.

In summary, our contributions include:

1. In user-level authentication, we introduce the idea of programmably leaving the result of authentication on the server (verifier). Such hiding of authentication results may enable effective protection against online guessing attacks.

2. We propose the use of adapted distorted image as a computer-cipher/human-decipher channel to communicate short messages in human-machine interaction.

3. Our proposal requires no changes on the client side software or existing password input UI, and can be used with any authentication scheme vulnerable to online guessing attacks.

\section{THREAT MODEL AND ASSUMPTIONS}

his section, we describe our goals, the conditions under Uvauth works, and list situations that are considered -scope.

; The objective of our proposal is to make both ne-only and human-assisted attacks significantly more lt than using the current state-of-the-art captchas. vel of difficulty can be set by the depth of deception uuth's fake sessions.

\section{nptions.}

er-level authentication. We address authentication ios where a human user is the claimant and a comis the verifier. We do not include machine-to-machine atication, e.g., automated script for connecting to a database server. 


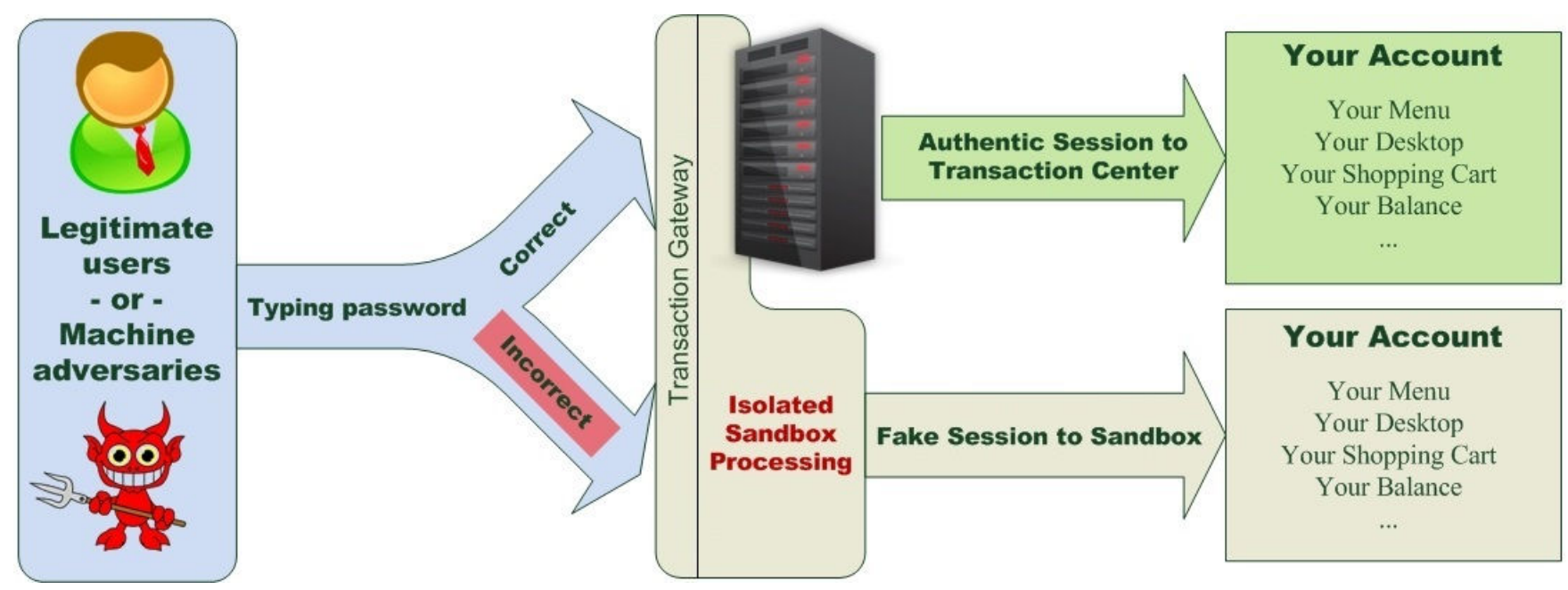

Figure 1: Overview of user-verifiable authentication

b) Weak-secret-based, single-factor authentication. Uvauth can be used independent of any existing authentication technique, e.g., text or graphical password schemes, certificate-based schemes. However, Uvauth's protection is intended for situations where weak-secrets are used that can be efficiently guessed through online attacks (e.g., a humanchosen password vs. a random 128-bit key). Multi-factor schemes that use an additional token or biometrics also may not need protection against guessing attacks, assuming the additional factors provide enough entropy. However, singlefactor multi-stage schemes (e.g., SiteKey or personal questions with passwords) may benefit from Uvauth; e.g., the fake session can start right at the end of first-stage of authentication. However, most of our discussion here considers only commonly-used single-stage password authentication.

c) Data-oriented sessions. We focus on accounts that mostly deal with user data (e.g., banking, email), instead of providing some generic services to the user (e.g., Internet access). Implementing fake sessions for service-oriented accounts could bn wito hollonmino if not impossible.

d) Separate $n$ no means of ac other than via $\mathrm{t}$. Otherwise, auth through side-che

e) Random at to be random in the user is knov may be detected file information,

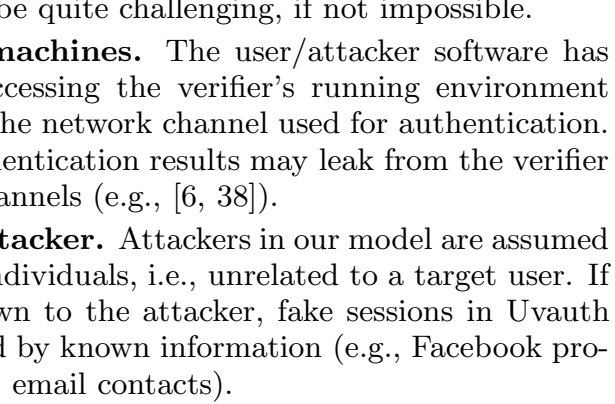

f) No offline attacks. We assume that data at rest is safe, e.g., password databases are inaccessible to attackers. Otherwise, simpler offline attacks can be mounted to reveal the passwords (if hashed or encrypted under a weak key).

g) Other password-unrelated security issues. Our proposal only addresses online password guessing; so, if a website or application is vulnerable to other types of attacks such as SQL injections, Uvauth's protection may not help. We also do not address several other threats, including: phishing, malicious software on the client or verifier, and session hijacking attacks.

\section{UVAUTH: USER-VERIFIABLE AUTHEN- TICATION}
In this section we discuss Ivauth and the underlying self- eviden $\quad$ lay make the scheme feasi- ble. By list of scenari
Overv
Legitin in tern accept also co user cr coperties, we also provide a fake sessions, and discuss nore applicable. rall architecture of Uvauth. ackers are treated equally, s. A transaction gateway on requests; the gateway is sers (e.g., it has access to ct userid-password pair is received, processing is handed over to the transaction center and a legitimate session is established. Otherwise, when the given password is incorrect, the user/attacker is redirected to a sandbox-enabled environment that hosts fake user sessions. The established sessions in both cases appear to be (almost) the same to a machine. A random human attacker may also be unable to judge the content of the fake account without performing some non-trivial tasks.

\subsection{Implicit detection of an authentication outcome}

We first distinguish the verifier sidered in get account where some

\subsubsection{Se}

If the dat and of relat be enough for a straightforward and effortless decision by the real data owner. In this case, the authentication result is implicit, i.e., requires no indication of failure or success. Consider the following as examples of this type of authentication. For most active users of a social networking site (e.g., Facebook), users can (possibly) easily identify their own accounts after a successful login-e.g., from the pro- 
file info, page layout, friends list and messages. The same is possibly also true for online banking login, identified by e.g., user info, account balance, transaction history and registered bills. These types of accounts are highly personal and quite unique to a user. More importantly, these accounts can be populated with fake information to make them indistinguishable even to non-owner human users (in addition to automated bots).

User-verifiability obviously requires that the same user experience is provided for a specific credential used. Therefore, to implement a user-verifiable authentication scheme that is both user-acceptable and attacker-indistinguishable, we must consider the following issues. First, each fake session generated for a specific userid-password pair (even if the userid is non-existent), must appear to be the same for a certain period of time. If randomness of fake sessions is distinguishable for login attempts with the same userid with different passwords, attackers can easily detect the difference, and then learn the authentication outcome. On the other hand, the fake session for a specific userid must change with time, as is the case for many user accounts (e.g., new messages and friends in a Facebook account; updated balance and new transactions in a banking account).

\subsubsection{Additional login help for legitimate users}

To aid users and help identify a successful login, a combination of the following methods can also be used.

a) Customized messages. A user customized welcome message may be used for the identification of a valid session. During account registration, a user can set up some personalized

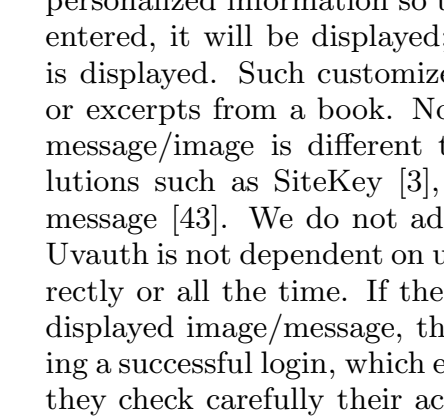

to known vulnerabilities in $\mathrm{S}$ cation secrets are leaked for $t$

b) Secondary channels.

SMS/twitter/email messages

a login is successful. Mobile $\mathrm{S}$

tus indication in many businesses, suc

card transactions (see e.g., MasterCar

assume here that the secondary chan mised; otherwise, an attacker can us verification. Periodically, users may a failed login attempts through secondi the form of a daily digest).

c) Warning messages. A warning message may be displayed so that the user is reminded that Uvauth is in place, and verify whether they can access the ir dnto message is as follows: "Please check yc case you do not see your expected datc correct password."

d) Dynamic security skins. Antisuch as synchronized random dynamic dynamic se tify an auth messages tc does not $\mathrm{re}$ always corr additional

\subsection{Designing fake sessions}

Uvauth's effectiveness depends on attackers being unable to detect fake sessions efficiently. Below, we discuss few considerations and account properties for designing effective fake sessions.

\subsubsection{Account properties}

Here we list four factors that may be used to categorize account types. We also discuss how these factors may be considered during the generation of fake sessions.

a) Server-side data retention. Here we consider whether the user is allowed to make changes after logged in and to what exte $\quad$ accessible when she logs back i ure of a user account could be $\mathrm{r}$ essions may also need to store at a changes are stored, inconsister useful to some extent; cf. Neagoe nly accounts (e.g., call logs of a pr ing fake sessions could be much $\epsilon$ he accounts generally allow at le le parameters). If the size of upc ost of consistent fake

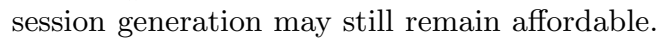

b) Client-side data representation. For most account types, users get access to some data after logged in. How much an attacker can understand the meaning of user data, determines how easily she can detect a fake session. For highly-personalized data (e.g., photos, blogs, and calendars), fake session detection would be significantly difficult for an attacker, even if human assistance is used; the attacker has no obvious means to distinguish between fake and real data. For impersonal, human-readable data (e.g., magazine subscriptions), fake sessions should be populated with contextaware, meaningful data. For impersonal data with machine semantics (e.g., protocol traffic or command responses), it may be more difficult to generate fake sessions, and sometimes specific restrictions should be applied to limit the cost of fake sessions (e.g., running processor-intensive jobs in a fake ssh session).

c) Update types. Some accounts are update-driven, i.e., frequently updated directly by both the account owner and thers for the purpose of communication; examples include email and social networking accounts. Some accounts are activity-driven, i.e., indirectly updated by user transactions; examples include credit card accounts. Some accounts may be of mixed type; e.g., a seller's Paypal account is updated by Paypal (e.g., transaction logs) and other users (e.g., comments). These different account types should be modeled correctly to design realistic fake sessions.

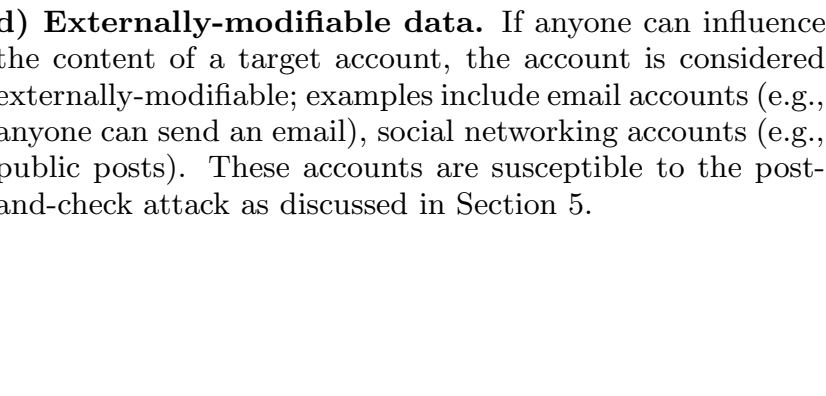




\subsubsection{Considerations for fake session generation}

a) Verisimilitude. There is a trade-off between the deployment of more realistic/consistent fake sessions with more functionality and resource consumption on the server. We define the depth of verisimilitude as the levels of operation a fake session would allow, before it may be detected by an automated attacker. Also, not all functions are equal in terms of costs-e.g., allowing the update of a profile parameter vs. searching for a transaction. As an example, consider a fake session at an online banking portal; an automated attempt can be performed by an attacker to transfer a cer-

account that directly/indirectly
ss are not far down in the oper-
fer-out is not activated for this
quired for this transaction". See
epth discussion on how to design
ers with a probabilistic model of
ever, we only want the effort for
oe non-trivial, instead of prevent-
ficant cost.

v) $1 \mathrm{~mm}$ m criaracterisucs. Sometimes due to network delay or processing on the server side, logging in or operations on a website are subject to different levels of responsiveness. Fake sessions should insert lags when required to simulate timing characteristics of different operations in the operations hierarchy, hours of the day, or even seasons in a year. This may also help confuse intruders as they cannot detect fake sessions by profiling timing characteristics. The freed time slots can be used for scheduling more fake sessions.

\section{DISTORTED IMAGE AS A COMMUNI- CATION CHANNEL}

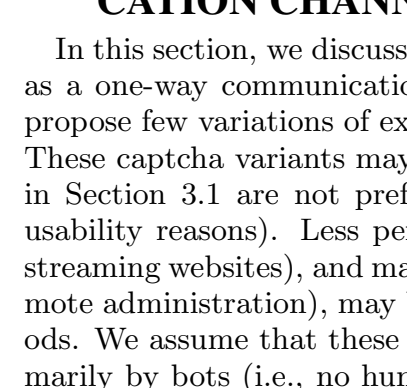

the possibility of using captchas on channel (server-to-user), and xisting captchas for this purpose. y be considered when techniques ferred (e.g., for deployability or rsonalized accounts (e.g., movie anaged-systems in batch (e.g., rebenefit from the proposed methaccounts would be attacked priman assistance), as they may be less valuable compared to personal/financial accounts.

\subsection{Captchas as a cipher}

Most current captcha techniques are based on the use of distorted images (or similar methods), and are used before authentication, to verify the presence of a human user. In contrast, we propose a post-authentication use of captchas. The idea is to utilize the generation and recognition of distorted images to communicate the authentication result back

(1)

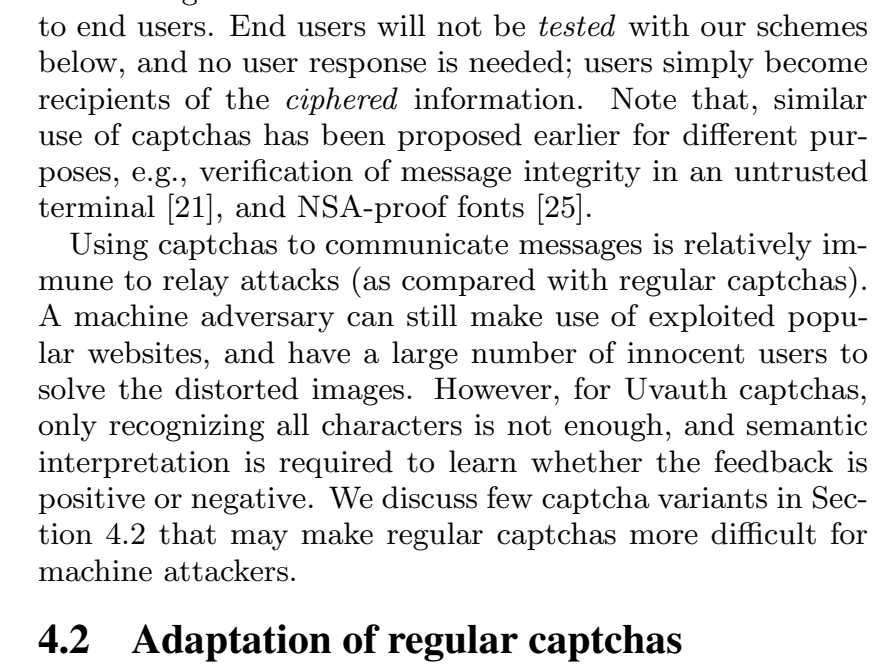

has, the content can $k$ $n$ irrelevant mix of letters and num rauth, we need to transmit messages s with predefined meanings for conve tion results. Existing captcha breaking tech: would perform even better against Uvauth the limited entropy of our messages (result the fixed nature of the messages). To : captcha generation may be adapted as foll a) Randomized padding. Humans have mantically interpret a message even if the $n$ to some extent. Most people do not read in a word, or even all the words in a senten As an example, consider the following sent is qKd k9l2 fine vMab weather " "us v You Msut Be Raelly Smrat"; in derstand the meaning without chines it is not straightforward these sentences, especially wher distorted image. As an example b) Indirect expression. Emo itive or negative expressions suc

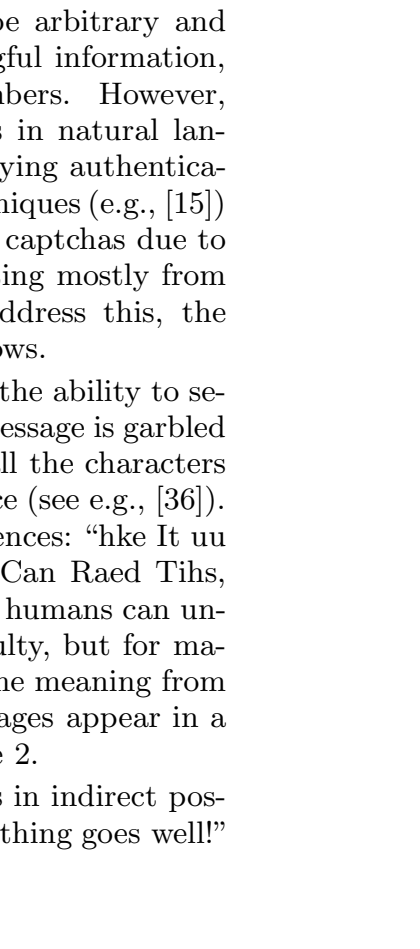




\section{It alfiject was angeco ighouf right connected}

Figure 2: Distorted image with random padding

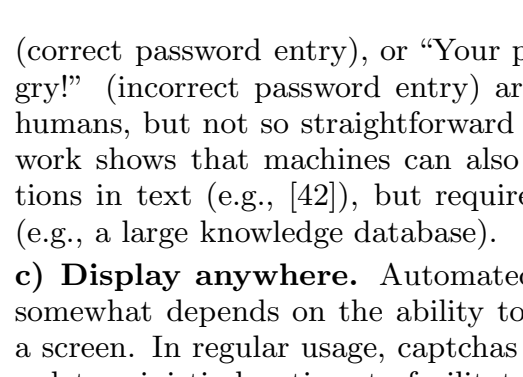

password makes me anrre quite self-evident for l for machines. Existing o learn to identify emores non-trivial resources

ed attacks on a captcha o locate the captcha on $\mathrm{s}$ are generally placed in a determminstic Iocalion, lo racintate the ease of processing by human users. As Uvauth's communication channel is one-way (i.e., no response back from the user), the distorted image can be placed anywhere on the screen (as long as it is visible to the user). It can also be embedded into a larger bitmap (e.g., banners, ads, backgrounds) to make automated identification difficult. Random delays (e.g., few seconds) may also be added before displaying tho mocaso (oftor tho authentication phase), to frustr

\subsection{An example with}

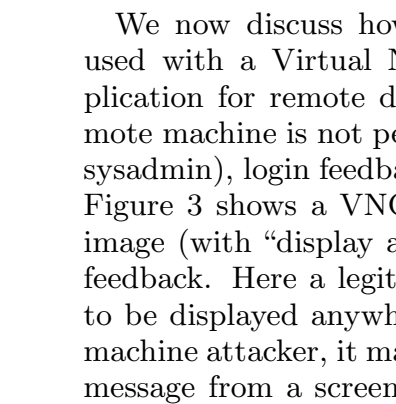
ow adapt
Network
desktop 1
personal t
oack via
C sessior
anywher timate $\mathrm{u}$ here on the screen. In contrast, for a nay be difficult to identify the distorted n-capture, specifically, when the message is blended with the background. Additionally, there is no need to display the distorted image right after login; e.g., a short, random delay can be added to confuse the attacker even further. The attacker may need to forward a video clip to a human solver to perform a relay attack, which would increase the cost of such an attack.

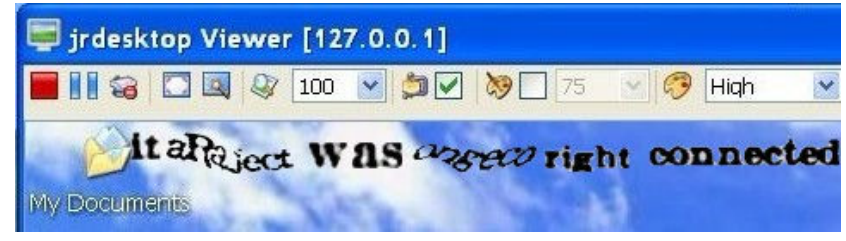

Figure 3: A VNC session with an adapted distorted image

\section{LIMITATIONS AND ATTACKS}

In this section, we evaluate Uvauth from an attacker's perspective and list possible attacks. Some of these attacks can be mitigated if special care is taken, while others are limitations of our current design.

a) Post-and-check attacks. For certain accounts, attackers can first post a message to the target account, and then check for the posted message when launching a guessing attack on that account. For example, an attacker can post a comment on the target's Facebook account, and by checking whether this specific post is seen, the detection of a fake session becomes easier. Similarly, an email can be sent to a victim's Gmail account for the same purpose, and the attacker then just checks whether the email has been received when in the fake session. We term these attacks as postand-check attacks, which can be automated and can make designing fake sessions significantly difficult. Applicationspecific defenses can be designed. For example, for a fake Facebook session, the target user's publicly-visible content, including posts from non-friends should be used. Assuming the attacker is not socially-connected to the user (i.e., not a Facebook friend), post-and-check attacks can be restricted.

Designing a similar mechanism for email is less straightforward, as no explicit social connections exist in email. However, email services (e.g., Gmail) are currently quite effective against spam email accounts; recently-received spam emails for a targeted account can be used in fake sessions albeit with the risk of some information leakage, as someimes legitimate emails are labelled as spam). Attackers also nust send an email to the target account immediately beore launching the guessing attack; otherwise, they would tot know whether the target user has deleted the unwanted mail, or they are in a fake session. Emails received from irst-time contacts in a recent period (e.g., in the last five ninutes) may be included in fake sessions. This can restrict ost-and-check attacks for email accounts, at the expense of ccasional information leakage.

v) Targeted attacks. If an attacker knows a victim in person (real-world or online-only), she may also know one or more contacts in the victim's Facebook friend list, or the accc online banking. When such

info

can

autc

(alt

nari

c) I

may

DoS

sess

to ove

So fak

activit

i.e., tho -1

sumption

d) Adapt

(e.g., as d

messages

such risk

tacker has

to the hum

e) Inconsistency attacks. II states saved, then the attacker may detect a some changes to it, and checking for re-login. This is a known problem in d as inconsistency of deception. Neagoe the attacker, a fake session us on restricting large-scale exclude targeted attacks be significant in some sce-

cks. Uvauth fake sessions rithmic/complexity-based sary can initiate many fake erations on the server-side earch in an email account. rofully, and the allowed too much resources; de and resource con-

ıuman solver services ed to attack Uvauth tas. We can alleviate here", so that the ata or even a video clip moffort

\footnotetext{
${ }^{1}$ jrDesktop, see
} 
that even inconsisten an attacker.

\section{f) Acquired target} attacker wants to gu $A$ and the attacker count $B$ from the sa word for $A$ is differen targeted attacks disc tracted information from $B$ to detect fake sessions for $A$. Note that, the attacker may need only temporary / onetime access to $B$. If the attacker can successfully guess the password for $A$, she can now use information from both acc accounts from the same user $\begin{array}{lr}\text { acc } & \text { accounts from the same user } \\ \text { ev } & \text { attack } \\ \text { ma } & \text { se of a } \\ \text { larg } & \text { t inci- } \\ \text { der } & \\ \text { g) } & \text { er suc- } \\ \text { cee } & \text { ssword } \\ \text { gue } & \text { sword, } \\ \text { e.g. } & \text { to the } \\ \text { leginimale user. Now, wrer } & \text { th the }\end{array}$ old password, he will be confused; by not seeing his data, the user might assume that he has mistyped the password, and keep trying several times before realizing the attack. Without Uvauth, the user will be denied access, and possibly try account recovery methods immediately.

A similar issue arises even when the account password remains uncompromised. If an incorrect password is tried (e.g., due to typos), users must detect the resulting fake session, and then log out for another attempt. word entries would cost more time for user: tional step of detecting fake sessions. This a side-effect of Uvauth, and does not happe feedback, as in regular authentication. No be avoided by displaying the password in $\mathrm{c}$. specifically when shoulder-surfing is not a user sitting alone in her office). However passwords may not be readily detected $b$ unmasking, and the user may still be delay the situation, partly due to Uvauth's fake

Other limitations include: we have not evaluated the serverside load for generating and running a large number of fake sessions. We also have not tested how effectively users can detect implicit results from an authentication attempt, or whether messages via adapted distorted images can be used in practice.

\section{REL}

Uvauth $\mathrm{f}$ deception te from both

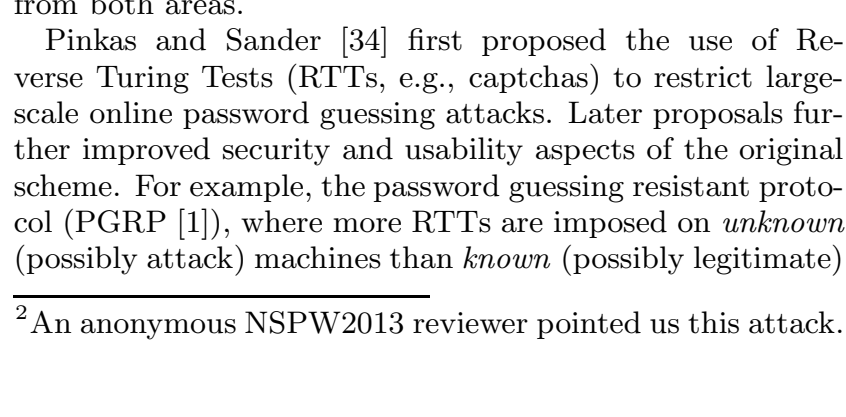

ones; machines are categorized using source IP addresses and $\mathrm{CO}^{1}$, chemes, Uvauth does not provi challenging us of distorted in ot require a re $n g$ paradigm (i: online passwi all) imposes a ent on each al idversely affe.o rogronnavo unoro ttempts are expected to be limitun. ... connews, nnu nunume may negatively impact an attacker when a large number of attempts are made from a single,$\quad$, It may not be effective agains

The idea of closely $m$ to distract/deceive ad lect analytical informa two decades of histor: ogy resembles honeyp also given deliberate a However, in cuses on hid: stead of dete the generatic a honeypot Provos desig. pots that sin level. It save

ypots), to cole than hodolcker is aation. ion foipt, inilar to the deployment of resource-intensive. $\mathrm{k}$ for virtual honeyzems at the network ; of resource consoleness. Additionally, ses, and thus allows emented. Uvauth's benefit from such existing hon-

e idea of security by obscurity, fenders, also have limited logical It is argued that the behaviour idden to gain tangible security of deception is limited to hiding ion outcome from attackers.

focuses on maintaining consispresented to attackers. Neagoe onsistent deception for defending rgue that these techniques may ack and monito make the desi resource-intens duce the cost o sessions in Uvauth.

Clark and Hengartner introduced panic where a separate password is used to indica ation to the server without soliciting an aut ure; the primary goal is to protect both th and sensitive information residing on the entry of a panic password, the observable $r$ ceive the adversary with panic responses that are indistinguishable from the real response. $\mathrm{W}^{\mathrm{r}}$ are proposed to be used by a legitim: Uvauth is targeted towards protectin ing guessed using a botnet, or by (rar attackers.

Juels and Rivest recently proposed 
passwords) to address offline attacks against hashed password databases. For each account, the legitimate password is mixed with several honeywords; thus, when an attacker cracks a hashed password, she cannot be sure if it is the real password or a honeyword. Also, the use of a honeyword will trigger an alarm on the server-side (cf. panic password).

\section{CONCLUSION}

We propose Uvauth to hide authentication results from attackers to mitigate the risk of online password guessing. It can effectively deceive an attacker assuming fake sessions can be efficiently generated (as an attacker may launch many authentication attempts from a large-scale botnet). Most current authentication schemes would fail to an adversary who is willing to use human help to break into existing techniques that are designed to limit only automated attacks. As user accounts generally become more and more valuable with the duration of use, it may be worthwhile for attackers to invest in cheap human labor as a means to compromise user credentials. In designing Uvauth, we explicitly consider such threats and provide limited protection (possibly significantly more than existing technologies). Implementing Uvauth fake sessions would require server-side support, but no changes are needed on the client-side software or existing password input UI (including browser mechanisms such as "keep me logged in" and cookies). However, Uvauth, as presented, has not been fully evaluated, and has a number of limitations. Our goal is to attract attention to the important drawback of authentication schemes that enables large-scale guessing attacks. We anticipate the vibrant discussion at NSPW will improve Uvauth, and help design better solutions to address online guessing attacks.

\section{Acknowledgements}

We thank our shepherd Michael Franz, anonymous NSPW2013 reviewers, Jeremy Clark, and members of the Concordia's Computer Security Lab for their insightful suggestions and advice. The second author is supported in part by an NSERC Discovery Grant and Concordia University Start-up Program.

\section{REFERENCES}

[1] M. Alsaleh, M. Mannan, and P. van Oorschot. Revisiting defenses against large-scale online password guessing attacks. IEEE Transactions on Dependable and Secure Computing (TDSC), 9(1):128-141, 2012.

[2] G. Bakos and S. Bratus. Ubiquitous redirection as access control response. In Annual Conference on Privacy, Security and Trust (PST'05), St. Andrews, NB, Canada, October 2005.

[3] Bank of America. SiteKey authentication: An

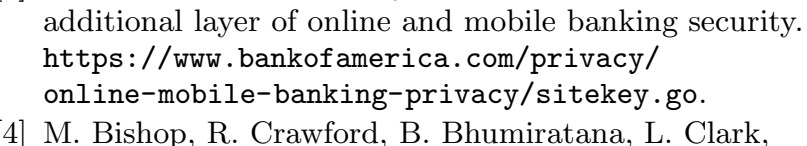
and K. Levitt. Some problems in sanitizing network data. In IEEE International Workshops on Enabling Technologies: Infrastructure for Collaborative Enterprises (WETICE 2006), Manchester, UK, June 2006.
[5] M. Bishop, J. Cummins, S. Peisert, A. Singh, B. Bhumiratana, D. Agarwal, D. Frincke, and M. Hogarth. Relationships and data sanitization: A study in Scarlet. In New Security Paradigms Workshop (NSPW'10), Concord, MA, USA, Sept. 2010.

[6] D. Brumley and D. Boneh. Remote timing attacks are practical. Computer Networks, 48(5):701-716, Aug. 2005.

[7] E. Bursztein, S. Bethard, J. C. Mitchell, D. Jurafsky, and C. Fabry. How good are humans at solving CAPTCHAs? A large scale evaluation. In IEEE Symposium on Security and Privacy, Oakland, CA, USA, May 2010.

[8] E. Bursztein, M. Martin, and J. C. Mitchell. Text-based CAPTCHA strengths and weaknesses. In ACM Computer and Communications Security (CCS'11), Chicago, IL, USA, Oct. 2011.

[9] B. Cheswick. An evening with Berferd, in which a cracker is lured, endured, and studied. In Winter USENIX Conference, San Francisco, CA, USA, Jan. 1992.

[10] J. Clark and U. Hengartner. Panic passwords: Authenticating under duress. In USENIX Workshop on Hot Topics in Security (HotSec'08), San Jose, CA, USA, July 2008.

[11] F. Cohen. The use of deception techniques: Honeypots and decoys. The Handbook of Information Security, 3:646-655, 2006.

[12] S. A. Crosby and D. S. Wallach. Denial of service via algorithmic complexity attacks. In USENIX Security Symposium, Washington, DC, USA, Aug. 2003.

[13] R. Dhamija and J. D. Tygar. The battle against phishing: Dynamic security skins. In Symposium on Usable Privacy and Security (SOUPS'05), Pittsburgh, PA, USA, July 2005.

[14] C. Dwork and M. Naor. Pricing via processing or combating junk mail. In Advances in Cryptology CRYPTO'92, Santa Barbara, CA, USA, August 1992.

[15] Futurity.org Cotahol montahn commitu Anws revealed. Arnilnhlont

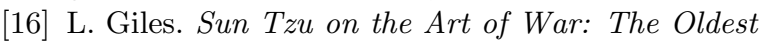
Military Treatise in the World. London Luzac, 1910. Chapter 1: verse 18.

[17] V. Goyal, V. Kumar, M. Singh, A. Abraham, and S. Sanyal. Compchall: Addressing password guessing attacks. In International Symposium on Information Technology: Coding and Computing (ITCC'05), Las Vegas, NV, USA, April 2005.

[18] T. Holz, M. Engelberth, and F. Freiling. Learning more about the underground economy: A case-study of keyloggers and dropzones. In European Symposium on Research in Computer Security (ESORICS'09), Saint Malo, France, Sept. 2009.

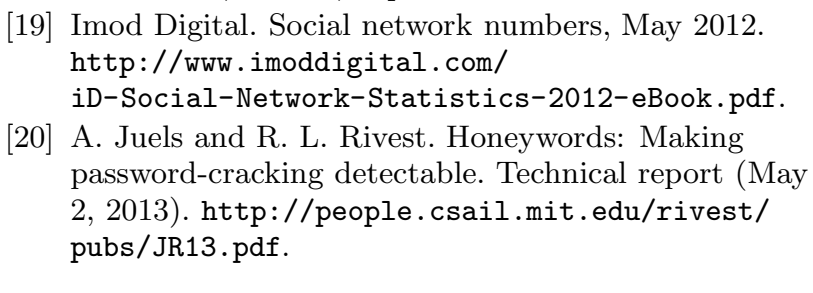


[21] J. King and A. dos Santos. A user-friendly approach to human authentication of messages. In Financial Cryptography and Data Security (FC'05), Roseau, Dominica, February 2005.

[22] Knowthenet.org.uk. More teenagers are being hacked

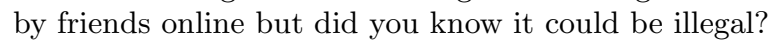
Norre nnticle (Jan. 6, 2012)

[23] vrasucruard. MasterCard inControl service now

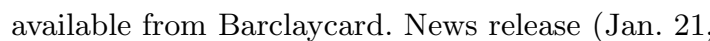
on1n)

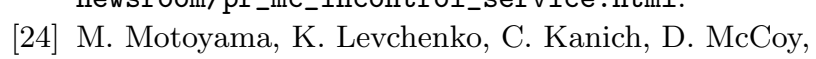
G. M. Voelker, and S. Savage. Re: CAPTCHAs understanding CAPTCHA-solving services in an economic context. In USENIX Security Symposium, Washington, DC, USA, August 2010.

[25] S. Mun. Making democracy legible: A dofinnt

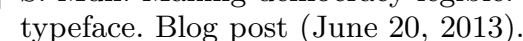
Project

[26] A. Narayanan anu v . Nmmaukov. Fast dictionary attacks on passwords using time-space tradeoff. In ACM Computer and Communications Security (CCS'05), Alexandria, VA, USA, November 2005.

[27] A. Narayanan and V. Shmatikov. Robust de-anonymization of large sparse datasets. In IEEE Symposium on Security and Privacy, Oakland, CA, USA, May 2008.

[28] V. Neagoe and M. Bishop. Inconsistency in deception for defense. In New Security Paradigms Workshop (NSPW'06), Dagstuhl, Germany, Sept. 2006.

[29] NewYorker.com. The inevitable downf 11

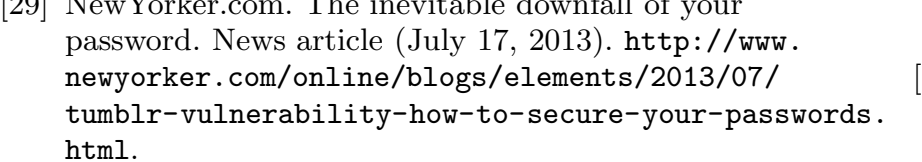

[30] J. Nielsen. Stop noncurnnd maclinn Anlinn anticle ( Twn no nחก)

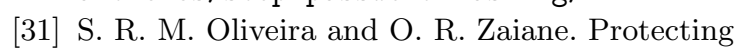
sensitive knowledge by data sanitization. In IEEE International Conference on Data Mining (ICDM 2003), Melbourne, FL, USA, November 2003.

[32] B. Parno, J. M. McCune, D. Wendlandt, D. G. Andersen, and A. Perrig. Clamp: Practical prevention of large-scale data leaks. In IEEE Symposium on Security and Privacy, Oakland, California, USA, May 2009.
[33] D. Pavlovic. Gaming security by obscurity. In New Security Paradigms Workshop (NSPW'11), Marin County, CA, USA, Sept. 2011.

[34] B. Pinkas and T. Sander. Securing passwords against dictionary attacks. In ACM Computer and Communications Security (CCS'02), Washington, DC, USA, November 2002.

[35] N. Provos. A virtual honeypot framework. In USENIX Security Symposium, San Diego, CA, USA, Aug. 2004.

[36] K. Rayner, S. J. White, R. L. Johnson, and S. P. Liversedge. Raeding wrods with jubmled lettres: There is a cost. Psychological Science, 17(3):192-193, Mar. 2006.

[37] T. Richardson, Q. Stafford-Fraser, K. R. Wood, and A. Hopper. Virtual network computing. IEEE Internet Computing, 2(1):33-38, 1998.

[38] T. Ristenpart, E. Tromer, H. Shacham, and S. Savage. Hey, you, get off of my cloud: exploring information leakage in third-party compute clouds. In $A C M$ Computer and Communications Security (CCS'09), Chicago, IL, USA, November 2009.

[39] N. C. Rowe. Designing good deceptions in defense of information systems. In the Annual Computer Security Applications Conferencen (ACSAC'04), Tucson, AZ, USA, December 2004.

[40] E. Shi, Y. Niu, M. Jakobsson, and R. Chow. Implicit authentication through learning user behavior. In Information Security Conference (ISC'10), Boca Raton, FL, USA, Oct. 2010.

[41] C. Stoll. The Cuckoo's Egg: Tracking a Spy Through the Maze of Computer Espionage. Doubleday, 1989.

[42] C. Strapparava and R. Mihalcea. Learning to identify emotions in text. In ACM Symposium on Applied Computing (SAC 2008), Fortaleza, Brazil, March 2008.

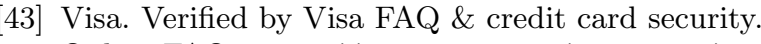
Onlino EAC

[44] L. L. 1E, N. NIIIU1, anu D. Amunony. Trusted paths for browsers. ACM Transactions on Information and System Security (TISSEC), 8(2):153-186, 2005.

[45] J. Youll. Fraud vulnerabilities in SiteKey security at Ronl of Amonion Tonhnionl antioln (Tulr 18, 2006).

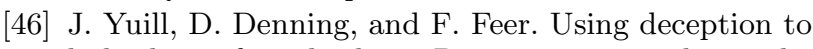
hide things from hackers: Processes, principles, and techniques. J. Information Warfare, 5(3):26-40, 2006. 